\title{
Notas sobre el uso de la antropología en el campo de la salud pública
}

\author{
Notes on the use of anthropology in the field of public health
}

Dr. Richard N. Adams†*

Reproducido del Boletín de la Oficina Sanitaria Panamericana.

Vol. XXXVIII, No. 5, mayo de 1955.

\section{NTRODUCCI ÓN}

El julio de 1951, el Dr. George Foster publicó un informe mimeografiado, redactado por él y basado en la labor llevada a cabo por los cuatro antropólogos del Instituto de Antropología Social de la Institución Smithsoniana. Este informe titulado "A CrossCultural Anthropological Analysis of a Technical Aid Program" (Análisis Antropológico Intercultural de un Programa de Ayuda Técnica), fue traducido al español y publicado en 1952 por el Instituto Nacional Indigenista de México, y el mismo año apareció un amplio resumen en el Boletín de la Oficina Sanitaria Panamericana, publicación que se distribuye en todos los países del Hemisferio Occidental. Dicho informe consistía en un análisis de la labor realizada por los Servicios del Instituto de Asuntos Interamericanos en ciertas fases de sus actividades en México, Colombia, Perú y Brasil. El estudio, como su título indica, trataba de analizar desde un punto de vista antropológico los esfuerzos del personal de salud pública para implantar, por medio de sus programas ordinarios, medidas de higiene y saneamiento en las poblaciones de regiones seleccionadas de la América Latina.

El informe de Foster no era, en modo alguno, el primero en que un antropólogo expresaba sus opiniones acerca de los métodos y programas de salud pública, pero fue uno de los primeros que trató de presentar un análisis sistemático de algunos de 
esos programas desde el punto de vista antropológico y formular recomendaciones basadas en dicho análisis encaminadas a cambiar las prácticas de salud pública. En ese aspecto marcó un jalón importante en el proceso de la aplicación de la antropología al campo de la salud pública, y produjo considerable impresión en muchos trabajadores de salud pública de la América Latina y de los Estados Unidos. A muchos de ellos les ofreció un excelente ejemplo de una de las formas en que la antropología cultural y social, como estudio de la conducta humana habitual, puede facilitar la labor de salud pública. Puesto que esta última se interesa fundamentalmente en modificar los hábitos humanos, los trabajadores en ese campo podrían beneficiarse de la ayuda prestada por las personas que se dedican especialmente al estudio de los hábitos humanos y de sus alteraciones. No cabe duda, sin embargo, que para muchos, el informe de Foster no pasó de ser un ensayo preliminar. La mayoría del personal de salud pública tenía escaso o ningún contacto con antropólogos culturales y no había tenido tampoco la oportunidad de observar en la práctica las formas en que la antropología podía secundar su labor. Además, el empleo de un antropólogo en los programas de salud pública era una idea tan nueva que pocos administradores estaban suficientemente convencidos de la utilidad de la disciplina o tenían los conocimientos necesarios sobre ella para fomentar su empleo en proyectos específicos de salud pública.

En los tres años transcurridos desde que apareció la publicación de Foster, los trabajadores de salud pública y los de antropología han tenido más ocasiones de descubrir la eficaz manera en que la antropología puede contribuir a la salud pública. En el curso de esos años, ha sido posible reevaluar, aclarar y complementar los principios e ideas propuestas por Foster. El presente trabajo tiene por objeto esbozar a la luz de la experiencia obtenida en los últimos años las distintas formas en que la antropología puede contribuir a la salud pública; estudiar la colaboración entre trabajadores de ambos campos de actividad en consonancia con el informe de Foster, y tratar de exponer lo que hemos aprendido desde su publicación. La discusión se circunscribirá a los siguientes límites generales: ¿En qué forma se puede utilizar con mayor eficacia la antropología para la mejor ejecución de los proyectos de salud pública? El punto de vista expuesto es el de una persona que, por haber trabajado en Centro América, no pudo examinar la mayor parte de la documentación relativa a la labor hecha en otras partes del mundo. Por consiguiente, este informe es parcial, tanto geográficamente como en cuanto al tiempo que abarca. Aunque existe un acopio cada vez más considerable de escritos sobre la materia, la mayor parte de ellos en manuscrito o mimeografiados, ha habido relativamente escaso interés en la manera en que la antropología ha contribuido al progreso de la salud pública, así como en los conocimientos obtenidos sobre la colaboración entre el antropólogo y los encargados de los programas de salud pública.

Las principales recomendaciones formuladas por Foster en 1951 pueden resumirse en las tres categorías principales siguientes:

1) Los administradores de programas de salud pública deben estar familiarizados con los valores culturales de la colectividad en que trabajan. Foster mencionó nueve aspectos sociales y culturales que el administrador debe conocer (e insistió en que no se trataba, en modo alguno, de una lista definitiva). Entre ellos, concedió especial importancia al conocimiento de las creencias relativas a las enfermedades y a su curación. Los otros aspectos son los recursos económicos de la población, la organización de la familia, educación e instrucción primaria, administración política, religión, sistemas de valores, complejo de prestigio y normas de acción.

2) Deben formar parte de los equipos de campo de los programas de salud pública especialistas de ciencias sociales, "para recoger informes especializados (para coronar un vasto acopio de datos fundamentales) tendientes a facilitar proyectos específicos". Además, tales especialistas pueden hacer continuos experimentos de campo variando 
los métodos en consonancia con diferentes condiciones con el objeto de determinar con mayor precisión la relativa eficacia de los mismos.

3) Los estudios fundamentales tanto sobre los rasgos culturales de una cierta región como sobre la teoría de la antropología, son esenciales para la eficacia de la labor del antropólogo. Foster señala que "... parece obvio que deben hacerse planes para proseguir la acumulación de datos culturales fundamentales de valor general no sólo en Latino América, sino también en otros lugares donde pretenda establecer programas de esta clase. Administrativamente, tales trabajos no se deben acoplar directamente con los programas de acción específica, porque la investigación enfocada hacia programas demasiado específicos da resultados de menor utilidad para otros tipos de programas."

Utilizando estos tres puntos como base de discusión podemos examinar algunos de los problemas y cuestiones que se plantean en la aplicación de la antropología al campo de la salud pública.

\section{LA ANTROPOLOGÍ A Y EL ADMI NI STRADOR DE SALUD PÚBLI CA}

De su conocimiento de la antropología y de su actitud hacia ella depende que el administrador de salud pública decida utilizarla o no dentro de su jurisdicción. Se puede afirmar que, en general, muy pocos de estos administradores tienen de esta disciplina más que los conocimientos más rudimentarios. Su actitud hacia la antropología va desde un excesivo entusiasmo que lo incapacita para delimitar los límites de sus posibilidades hasta una declarada hostilidad, pasando por una total indiferencia. Rara vez pudo el administrador de salud pública hacer una valoración crítica de la antropología o definir los linderos de sus aplicaciones partiendo de su experiencia personal.

El entusiasmo exagerado por la antropología lleva, por lo general, a suponer que puede lograr "lo imposible", o que puede producir cambios que requieren años en cuestión de semanas. La hostilidad se manifiesta, de ordinario, en el médico o trabajador de salud pública, que pretende saber ya más de lo que un antropólogo puede llegar a saber de una cultura dada. Uno se encuentra a veces con un médico o con un profesional de salud pública que, por el hecho de ser del país, pretende saber más sobre el medio cultural en que vive de lo que pueda llegar a saber cualquier antropólogo extranjero. La falta de base de semejante argumento se pone de manifiesto suponiendo que un enfermo que pretendiera saber cómo tratar su enfermedad mejor que el médico, ya que éste, al fin y al cabo, no la padece. Sin embargo, la mayoría de los funcionarios de salud pública están tan poco familiarizados con las posibilidades que la antropología ofrece que ni se muestran entusiastas ni hostiles a ella, sino simplemente curiosos.

El interesar en la antropología a los administradores de salud pública no es un problema de propaganda ni de abrir canales de comunicación a fin de que las ideas antropológicas puedan llegar al trabajador de salud pública. La antropología no trata de convencer de su utilidad a los medios responsables de la salud pública; tampoco se logrará una comprensión efectiva de esta ciencia por el mero hecho de que se faciliten informes antropológicos a los administradores. En los cuatro años de asociación del autor de este trabajo con actividades de salud pública ha visto circular artículos y documentos sobre antropología entre el personal de esta especialidad. Mientras los leía trataba de imaginarse la situación del administrador de salud pública a cuyo despacho llega diariamente una gran cantidad de correspondencia y documentos, y se ha preguntado: ¿Qué efecto puede tener un artículo de antropología en un 
administrador que se encuentra agobiado por la preparación del presupuesto del año próximo y por el problema de uno de los automóviles de la oficina que acaba de tener un accidente $y$, por alguna inadvertencia, no estaba asegurado? Además, cuando disponga de tiempo libre, lo dedicará a la lectura de publicaciones sobre alguna materia de interés especial o de una novela como descanso o recreo. No obstante, es cada vez mayor el número de administradores que llegan a leer tales artículos e informes debido a que, en la ejecución de sus programas, se han encontrado con problemas y han llegado a creer que la antropología puede ofrecer soluciones a algunos de ellos.

El problema principal que se plantea al presentar la antropología al administrador es, de algún modo, suscitar en él cierto interés en la materia, por lo menos en grado suficiente para incitarle a enterarse de sus posibilidades, y una vez interesado, buscar algún medio de que adquiera algunos conocimientos sobre esta ciencia. Es indudable que la mejor ocasión de estimular el interés por la antropología es el período de formación profesional, durante los cursos de licenciatura o doctorado en salud pública. Algunas escuelas de salud pública se han preocupado de proporcionar este estímulo. La Fundación Russell Sage ha establecido algunos puestos de profesor de antropología en las Universidades de Harvard y Denver con esta finalidad. La Universidad de Cornell enseña cursos de esta materia para el personal de salud pública y la Escuela Nacional de Salud Pública, de México, cuenta entre su personal docente con un antropólogo empleado a tiempo parcial. Sería conveniente examinar aquí en detalle el problema de brindar esta formación en las escuelas de salud pública, pero las actividades del autor no se han orientado hacia ese campo y, por lo tanto, lo deja a personas con mayor experiencia pedagógica. No obstante, subsiste un problema de suma importancia. La Mayoría de los actuales administradores de salud pública no estudiaron antropología durante sus cursos de salud pública; algunos nunca siguieron cursos de salud pública ni de antropología. Por consiguiente, no sólo ahora, sino durante muchos años, habrá que inculcar las ciencias sociales a las personas que trabajan a tiempo completo en la administración de salud pública.

La inclinación a utilizar las ciencias sociales en las actividades de salud pública puede suscitarse de una o de ambas maneras siguientes: 1) insistiendo cerca de los administradores hasta que manifiesten suficiente interés para intentarlo y, 2) demostrando en la práctica que la antropología, en realidad, puede solucionar problemas difíciles o imposibles de resolver sin ella. Las relaciones que la mayoría de los administradores mantienen no comprenden a muchos antropólogos, y aun cuando de palabra llegara a suscitar su interés, siempre podrían desear una demostración. En resumen, para que un administrador se familiarice con la materia, ha de ver la antropología en acción, y para verla hay que contar con algunos administradores convencidos de su utilidad. Todo ello indica que transcurrirá largo tiempo antes de que logremos que muchos administradores se familiaricen, bien sea con la antropología o con los medios de llegar a conocer el medio cultural en que trabajan. Evidentemente, tiene suma importancia estimular el uso de la antropología siempre que resulte eficaz, a fin de que su utilidad se puede apreciar mejor.

\section{ESPECI ALI STAS EN CIENCI AS SOCI ALES EN LOS PROYECTOS DE CAMPO}

La recomendación de Foster de que se utilicen especialistas en ciencias sociales en los programas de salud pública era ya seguida cuando su informe apareció. Ahora bien, la labor llevada a cabo desde entonces ha permitido determinar mejor la función que los antropólogos pueden desempeñar con eficacia en el campo de la salud pública. A continuación vamos a examinar el tipo general de trabajo que la antropología puede 
llevar a cabo, así como ciertas actividades específicas: investigación, consulta y enseñanza.

\section{Campos de la salud pública en los que la antropología puede ser de utilidad}

Una de las dificultades que algunos antropólogos adscritos a actividades de salud pública han tenido que afrontar es que, por lo general, el personal de salud pública tiene un concepto tan superficial de la antropología, que no se hace idea de la manera más efectiva de valerse de ella para sus fines. Con el objeto de mantener cierto orden administrativo en los trabajos en ejecución, a veces ha habido la tendencia a encajar al antropólogo en el cuadro burocrático de la organización de salud pública, limitando así en grado considerable la eficacia de esta aportación de las ciencias sociales.

El hecho de que una determinada materia corresponda a cierto campo de la salud pública ni afirma ni niega, por sí solo, que la antropología pueda ser de utilidad en tal caso. Cabe decir, en general, que la posible utilidad de la antropología puede juzgarse únicamente en relación con los problemas específicos que se plantean (o que puede preverse que se van a presentar) al iniciar una actividad específica de salud pública en determinadas circunstancias culturales, sociales y de ambiente. Por ejemplo, la organización de un programa de control de la malaria, un nuevo sistema de abastecimiento de agua, una escuela de enfermería o una clínica rural, puede o no beneficiarse del uso de la antropología, según sea la naturaleza de los problemas específicos del caso. En lugares donde se sabe que la malaria se debe a la presencia de mosquitos de cierto tipo, tal vez sea suficiente la aplicación de los métodos corrientes para la ejecución de los programas sin embargo, cuando se cree que la enfermedad se debe a enfriamiento después de haber sudado, tal vez sea necesario utilizar recursos más complicados. En circunstancias muy difíciles, será conveniente contar con los servicios de un antropólogo que pueda interpretar el cómo y porqué de las creencias tradicionales locales. Muchas colectividades en todos los países del mundo verían con sumo agrado que se mejorase su abastecimiento de agua; sin embargo, cuando una colectividad tiene creencias peculiares respecto a la procedencia del agua, tal vez sean útiles los servicios de un antropólogo. A primera vista la creación de una escuela de enfermería no es la clase de empresa en que se considere conveniente la colaboración de un antropólogo. Ahora bien, cuando en el país en que se va a establecer la enfermera ocupa una categoría social baja, el encontrar alumnas puede constituir un verdadero problema. Del mismo modo, el hecho de que la organización de una clínica rural se beneficie o no de los servicios de un antropólogo depende de las características culturales y del medio en que se trata de establecer la clínica; también puede depender de la naturaleza de la organización del proyecto que va a establecerla.

Como ya se ha mencionado anteriormente, no es posible predecir de un modo definitivo, basándose únicamente en la naturaleza del proyecto, si será o no necesario emplear las ciencias sociales. Ahora bien, existen algunas indicaciones que muchas veces pueden ayudar a adoptar una decisión previsora**. Cabe citar las siguientes: a) cuanto mayores sean las diferencias culturales entre el personal encargado del proyecto y la población a que beneficia; b) cuanto mayor sea la distinción de casta o clase social entre el personal y la población, de mayor utilidad serán las ciencias sociales; c) en aquellos proyectos relacionados con alteraciones de los hábitos humanos que se adquieren en las primeras fases de la vida o se refuerzan a menudo, los servicios de un antropólogo o psicólogo social serán útiles. Estos no son los únicos casos en que la labor de los especialistas en ciencias sociales ha sido de utilidad, pero si tales situaciones existen, es indicación evidente de que los servicios de ese especialista serán una valiosa adición al proyecto. Los médicos, enfermeras y sanitaristas, por su adiestramiento y contactos personales de carácter profesional, tienden a establecer ideas comunes que pueden muy bien no ser compartidas por 
otras personas que pertenecen a su mismo grupo social y cultural. En ese aspecto, difieren en cultura de aquellos a favor de quienes habrán de trabajar. Aun suponiendo que lo contrario sea cierto, conviene recalcar que la mera existencia de un nivel educativo satisfactorio en la población no constituye, por sí sola, una indicación de que la cultura de la población general sea similar a la del personal del proyecto.

De acuerdo con los puntos antes mencionados se pueden indicar ciertos tipos de población en los que los proyectos puede la utilización de las ciencias sociales, a saber: a) En general cualquier sector de una población que, en su conjunto, es predominantemente de bajo nivel económico y social, puesto que la cultura de esa población, de ordinario, varía manifiestamente de la de las autoridades médicas de dicho país; b) Las poblaciones indígenas o de origen cultural extranjero, bien constituyan una mayoría o una minoría de la sociedad en conjunto, pueden diferir en costumbres de la población local dominante de que procede el personal médico; c) los proyectos encaminados a mejorar la nutrición y a cambiar hábitos fundamentales de higiene y saneamiento afectan a hábitos muy arraigados en la gente y estrechamente relacionados con muchas actividades de la vida cotidiana. No es fácil cambiar estas actividades. En términos generales, cabe decir que la antropología puede prestar ayuda específica en los programas de nutrición, educación sanitaria; higiene materno infantil, ciertas actividades de saneamiento del medio y en el establecimiento de programas integrados de salud pública. En términos más generales, puede contribuir a orientar los principios fundamentales de tales programas así como a la labor de investigaciones específicas.

\section{El papel de la investigación antropológica en la salud pública}

La antropología ha sido, tradicionalmente, una disciplina de investigación. Hasta los últimos años no pasó activamente al campo de la aplicación y, por consiguiente, su función en ese aspecto está todavía en período de desarrollo y definición. Algunos antropólogos, por razón de su personalidad, rinden también servicios eficaces en el campo de aplicación práctica, y una escuela de los Estados Unidos está fomentando un campo que denomina "antropología de acción", una combinación de trabajo práctico y de investigación. Sin embargo, en opinión del autor, la verdadera contribución del antropólogo descansa en su capacidad de investigador y consultor. Pocos antropólogos dominan las técnicas generales de educación, están familiarizados con actividades especializadas de grupo, con la dirección y orientación de adultos o con trabajo social que les permita desempeñar un papel particularmente eficaz en el campo de aplicación de su especialidad; por consiguiente, han de actuar como "amateurs" en ese campo. En las actividades llevadas a cabo por el autor, su equipo de campo contaba con algún personal cuyo objeto era poner en práctica los resultados de la investigación a medida que se iban conociendo.

Hay ciertamente ocasiones en que el antropólogo puede ser, a largo plazo, de mayor utilidad en relación con un proyecto si se abstiene de participar en persona en las actividades de ejecución y sigue sirviendo en su capacidad de investigador. Esto, por supuesto, varía de acuerdo con las características del programa, con la sociedad de que se trate y con el propio antropólogo.

Si se admite que la investigación es una importante contribución del antropólogo a la salud pública, vale la pena definir con mayor precisión la naturaleza de la misma y explorar sus posibilidades en relación con programas de aplicación práctica. En primer lugar, hemos de definir tres tipos generales de investigación que el antropólogo puede llevar a cabo: investigación de problemas, investigación de exploración e investigación de aplicación experimental. 
La investigación de problemas: es la encaminada a la solución de un problema específico que se presenta en el curso de un programa de salud pública. Por ejemplo, si un programa progresa con lentitud debido a la falta de colaboración de la colectividad, el antropólogo puede tratar de hallar sus causas y de este modo, contribuir a poner fin a este estado de cosas. Si un proyecto tiene por objeto adiestrar parteras empíricas en una comarca o país donde la obstetricia científica jamás se puso en práctica, el antropólogo puede estudiar la situación de las parteras locales y tratar de prever los obstáculos que la relación con ellas puede plantear a la ejecución del proyecto. Parte de la labor más eficaz llevada a cabo hasta ahora por los antropólogos en el campo de la salud pública ha sido en este aspecto de la investigación de problemas o en relación directa con él. En general, se puede definir el papel del antropólogo en la investigación de problemas como el que trata de resolver algún problema específico que obstaculiza la ejecución de un proyecto.

Investigación de exploración: por investigación de exploración se entiende aquí la región que se lleva a cabo en una región geográfica o en un grupo de población poco conocido generalmente con el objeto de determinar de una manera más o menos detallada el estado cultural, social y económico de los habitantes a fin de planear el programa de salud pública correspondiente de acuerdo con sus necesidades y posibilidades. Las materias objeto de esa exploración dependen del tamaño y complejidad de la sociedad, del tiempo disponible para la investigación (sin olvidar el tiempo de aprender su idioma, si fuera necesario), y de los aspectos específicos de la vida que puedan ser afectados por el mencionado programa. Puesto que el objeto de un estudio de esta clase es determinar los aspectos culturales que pueden oponer obstáculos o merecen ser tenidos en consideración al planear un programa, el antropólogo ha de emprender a menudo su investigación sin saber concretamente cuáles son los objetivos que persigue. Únicamente puede tratar de sondear las posibilidades, basándose en su formación y experiencia. Esto supone tener que realizar una investigación general que comprenda la economía, la estructura de la colectividad, organización de la familia, otras fases de la organización social y el sistema de valores, con el objeto de tratar de obtener una idea general de la manera en que los miembros de la colectividad reaccionan entre sí y a personas extrañas, y la forma en que reaccionarían ante los diversos factores que un programa de salud pública pueda introducir en sus colectividad.

La investigación exploratoria tiende a evitar que más tarde se planteen problemas, a facilitar el planeamiento con suficiente inteligencia de forma que requiera un mínimum de investigación de problemas. No puede planearse, con anticipación, de una manera minuciosa por la sencilla razón de que el antropólogo, a menos que esté familiarizado con medios culturales similares, no puede saber exactamente qué es lo que busca, aparte del hecho de que se trata de identificar aspectos de la cultura que más tarde puedan asumir especial significación en alguna fase de las actividades del proyecto.

Investigación de aplicación experimental: por investigación de aplicación experimental entendemos la encaminada a precisar la importancia o el valor de procedimientos, técnicas o métodos específicos en las actividades de salud pública, mediante la utilización de determinadas situaciones naturales como campos de experimentación y variando ciertos factores con el objeto de observar la manera en que afectan los resultados. Teniendo en cuenta los problemas que se han presentado en los proyectos de salud pública en América Latina, este tipo de investigación puede ser de suma utilidad. La educación sanitaria es un campo que puede beneficiarse especialmente de esta labor experimental. Abunda en técnicas heredadas del trabajo social, de la educación y de otras disciplinas más o menos establecidas que se han desenvuelto en las culturas occidentales. En la transferencia de esas técnicas a otros medios culturales y a otras formas de sociedad, se persiste a menudo en utilizarlas a pesar de que hay claras indicaciones de que distan mucho de ajustarse bien a la contextura 
cultural en que operan. Tal vez los resultados evidencian que esa labor de educación sanitaria no es satisfactoria, pero el único medio de encontrar procedimientos mejores es llevar a cabo experimentos en situaciones prácticas.

Es evidente que los tres tipos de investigación mencionados no se excluyen mutuamente. Una de las contribuciones más eficaces que la antropología puede aportar a un proyecto consiste en proceder en primer lugar a la investigación exploratoria, seguida de investigaciones experimentales, contando además en todo momento con personal para la investigación de problemas a medida que se plantean.

En la práctica, la labor de los antropólogos, por diversas razones, se ha limitado por lo general a uno de esos tipos de investigación. El trabajo de Erasmus, Simmons, Oberg, Kelly y Foster, todos ellos miembros del Instituto de Antropología Social, fue principalmente de investigación de problemas. La labor más reciente de Kelly, con el Instituto de Asuntos Interamericanos, ha sido de carácter más exploratorio, en tanto que Erasmus tuvo ocasión de llevar a cabo en el Ecuador algunas investigaciones experimentales en educación sanitaria. El grado en que las diferentes clases de trabajo se llevan a cabo depende hoy casi exclusivamente de la decisión del antropólogo; pocos administradores de salud pública se han percatado de la variedad de actividades que pueden desarrollarse.

La labor de investigación del antropólogo se puede considerar también en relación con la materia a que se aplica. Es conveniente tratar esta cuestión bajo tres aspectos generales: concentración en la organización que patrocina las actividades, y concentración en las relaciones entre la colectividad y la organización.

\section{Estudio de la colectividad}

Este ha sido hasta ahora el uso más aceptable y eficaz de la antropología en la América latina. Muchas personas que trabajan en el campo de la salud pública han visto la antropología en acción, investigando las causas por las que una población dada ofreció resistencia a un cambio, por qué reaccionó como lo hizo ante un proyecto, por qué prefería una clínica a otra, etc. El hecho mismo de que la antropología se ocupe por lo general de las sociedades aborígenes o no occidentales facilita la comprensión de su utilidad por parte de las personas no familiarizadas con esta disciplina en el estudio de esas sociedades. Sin embargo, el hecho de que el estudio antropológico de una colectividad sea o no provechoso no depende únicamente de que forme parte de una de las categorías clásicas de estudio antropológico. En los últimos años, la antropología ha logrado algunos de sus más notables adelantos mediante el estudio de las sociedades occidentales actuales. Se ha visto repetidamente que la persona perteneciente a una colectividad y de la misma cultura que la generalidad de sus miembros puede ser incapaz de comprender su propia sociedad, a menos que la estudie de un modo más o menos sistemático. Ahora bien, tal vez el $99 \%$ de las personas carecen de los medios, de la aptitud o del deseo de estudiar sistemáticamente su propia sociedad y por consiguiente, actúan como parte de ella sin percatarse de los muchos factores que operan en el seno de la misma. Desde este punto de vista, la antropología puede también contribuir en grado considerable al estudio de cualquier grupo social de hoy en que se trate de llevar a cabo un programa de salud pública. Sin embargo, a medida que se aleja de este campo de estudio clásico, la antropología tiende cada vez más a fundirse con el campo general de las relaciones y la conducta humanas, amplia disciplina a la que contribuyen las diversas ciencias sociales, psicológicas y biológicas. Los estudios de una dada sociedad pueden abarcar un amplio campo de investigación, tan amplio como la gama de aspectos de la sociedad misma. Tales estudios, como ya se ha mencionado anteriormente, pueden converger en un problema, o bien ser de carácter exploratorio o experimental. 


\section{Estudio de la organización}

El autor, entre otros, ha observado que la organización encargada de preparar y llevar a cabo un programa de salud pública suscita a menudo graves problemas. Estos proceden con frecuencia de la organización social del grupo que administra el programa. Según sea la estructura de dicha organización, la rapidez en sus decisiones será mayor o menor; la comunicación entre el personal esencial se facilitará o paralizará completamente; el personal está relativamente libre, o por el contrario, sujeto constantemente; el personal estará relativamente libre, o por el contrario, sujeto constantemente a fuerte presión psicológica; la autocrítica objetiva puede ser orientada adecuadamente o bien eliminada por completo, en resumen, de cómo esté organizado el personal de salud pública depende que un proyecto encuentre continuas dificultades hasta terminar en un rotundo fracaso, o bien tenga posibilidades de éxito.

Citaremos un ejemplo análogo: Durante los últimos años algunas escuelas de administración de negocios de los Estados Unidos han incluido en su plan de estudios asignaturas enseñadas por psicólogos sociales y por especialistas en actividades de grupo, con el objeto de que el futuro hombre de negocios tenga un mejor conocimiento de los factores sociopsicológicos que pueden contribuir a que la organización de una empresa comercial funcione normalmente y rinda ganancias, o a que no prospere debido a constantes dificultades de origen personal, con las consiguientes pérdidas económicas. Cabe suponer que también el administrador de salud pública desea obtener "ganancias", expresadas en éxitos de las actividades de salud pública. El administrador de una organización deficientemente estructurada puede encontrarse con que el éxito de su proyecto corre exactamente el mismo peligro que el negocio cuya estructura orgánica es defectuosa.

Este es un campo en el que los antropólogos no han figurado como dirigentes y orientadores, pero en el que los conceptos y métodos antropológicos son de suma utilidad. Los que han Ilevado a cabo estos estudios han trabajado por lo general en el campo de la investigación de comunicaciones, de las relaciones industriales y de la psicología social. Un sociólogo familiarizado con la labor en estos campos, puede determinar con frecuencia las causas de las dificultades experimentadas en la administración de un proyecto cuando el administrador y demás personal del mismo desconocen totalmente la causa de su mal funcionamiento. Esto es especialmente cierto en los proyectos en que trabaje personal docente de diversos países y de distintos antecedentes culturales.

\section{Estudio de las relaciones entre Organización y la sociedad que aspira a servir}

Cuando un proyecto de salud pública comienza a funcionar en una sociedad dada, pueden encontrarse (desde el punto de vista antropológico) dos concepciones diferentes; es decir, por lo menos dos grupos de personas de creencias y hábitos distintos han entrado en contacto directo, de persona a persona, y uno de esos grupos desea modificar las ideas y hábitos del otro. El caso más obvio y extremo de las diferencias es aquel en que un equipo de personal médico, sanitario y de enfermería, de formación occidental y habituado a la vida de ciudad, aparece en una colectividad indígena de México, Bolivia o Brasil, donde ni siquiera pueden hablar la lengua local. La mayoría de los trabajadores de salud pública estarán probablemente de acuerdo en que, en una situación semejante, sería de utilidad contar con los servicios de un antropólogo debidamente capacitado. Sin embargo, muchas veces no se tiene en cuenta que el personal médico, sanitario y de enfermería posee unas características culturales que le distinguen de los otros miembros de su respectiva sociedad occidental. Cuando un empleado de salud pública inicia o promueve un cambio de cualquier orden en una colectividad, indica con ello que algunos de sus 
puntos de vista o principios deben ser aceptados por ella. En cierto modo es lo mismo que se trate de la colectividad en que el médico nació o de un grupo socio-cultural totalmente diferente. En ambos casos, una persona que cree en un principio, o lo pone en práctica, desea imponerlo a un grupo de personas que no lo practican. Por lo tanto, ya sea que el oficial de salud pública ejerza en su propia ciudad o en una colectividad distinta, trata de hacer la misma cosa: introducir alteraciones en un sistema de hábitos arraigados en la sociedad.

En las relaciones entre la organización de un proyecto y la sociedad a que se destina, se plantea un problema importante por el hecho de que los miembros de cada grupo observan la conducta de los del grupo opuesto y los juzgan fundándose en las características manifiestas de esa conducta. El personal de salud pública de antecedentes y formación occidentales sabe cómo un médico de salud pública ha de actuar en nuestro propio medio social, pero son pocos los que saben comportarse en una colectividad india que habla una lengua indígena. Tal vez a un indio le extrañe bastante que el médico actúe como si estuviera haciéndole un favor cuando él estima que le hace un favor al primero por el mero hecho de llevar a su familia para ser vacunada. El agente de salud pública que en una colectividad clausura un restaurante por sus condiciones antihigiénicas, probablemente no será objeto de agradecimiento. Esto nos lleva a un problema fundamental que se plantea en las relaciones entre el personal de salud pública y los miembros de la colectividad: se trata del problema de predecir la conducta. En la medida en que el agente de salud pública puede predecir la conducta de los miembros de la colectividad, puede adaptarse su método con el objeto de lograr el mayor éxito en la consecución de sus fines profesionales. Del mismo modo, en la medida en que los miembros de la colectividad afectada pueden predecir la conducta del agente sanitario, podrán comprender lo que él desea y adaptar su conducta para secundar dichos deseos o para combatirlo. Ahora bien, cuando esta predicción resulta imposible, la respuesta del uno al otro puede tener escasa relación con las actividades u objetivos que el agente persigue.

Para citar un ejemplo más concreto, el presente trabajo tiene por objeto explorar algunas de las relaciones que pueden existir entre los antropólogos y el personal de salud pública, dos tipos de personas pertenecientes a grupos culturales diferentes dentro del marco de la sociedad occidental. Tiende a lograr que los trabajadores de salud pública puedan predecir con más facilidad la conducta de los antropólogos. Cierto grado de predicción es requisito esencial para el trabajo en colaboración.

Otro punto de alguna importancia para utilizar las ciencias sociales en la salud pública es la incorporación de esas actividades de investigación en el planeamiento y estructura general de un proyecto de salud pública. Hasta ahora hemos hablado casi indistintamente de "antropología" y "antropólogo". En realidad, puede haber bastante diferencia en el empleo de la antropología por un lado y un antropólogo, por el otro.

Aunque en una época el autor era de la opinión de que la prueba definitiva del éxito del antropólogo en un proyecto era su auto-eliminación, es decir, el hecho de resolver tantos problemas y capacitar a tantas personas que ya no quedaran problemas por resolver ni personas por capacitar, ahora está convencido de que este punto de vista es erróneo. El antropólogo es un especialista en cierto tipo de trabajos. Algunos de ellos pueden encomendarse sin dificultad a otras personas y enseñarse a otros especialistas. Ahora bien, no todos los problemas de salud pública pueden resolverse con facilidad. Si así fuera, no habría necesidad de contar con los antropólogos. EI hecho es que, a menudo, esos problemas requieren que el antropólogo aporte toda su formación y posibilidades para su solución; no se puede capacitar fácilmente en breve tiempo a especialistas en otros campos para que puedan resolverlos. 
Tal vez el procedimiento más práctico de utilizar la antropología sea facilitar a los médicos, enfermeras, sanitaristas, etc. Suficientes conocimientos en esta materia y en relaciones humanas a fin de que puedan por sí mimos beneficiarse de sus enseñanzas. Esa formación, especialmente si se refuerza con el trabajo práctico en casos individuales, puede ayudarlos a resolver por sí mismos muchos de los problemas que se planteen y permite al antropólogo asignado a un proyecto concentrar sus esfuerzos en aquellos otros que, por su grado de dificultad no está capacitado para resolver otro personal especializado. De este modo, se requerirían menos servicios del antropólogo y se depararía al personal del proyecto a satisfacción de haber resuelto sus propias dificultades.

Sin embargo, este ideal de contar con especialistas de salud pública versados en los fundamentos de la antropología dista mucho de ser un hecho y, por lo tanto, no obraría con criterio realista el que basara en esa posibilidad el planeamiento para el futuro inmediato. En la prestación de servicios, el antropólogo seguirá encargándose, durante algún tiempo de llevar a cabo la mayor parte de las investigaciones sobre los problemas sociales y culturales de proyectos específicos.

Además de las distinciones entre los diferentes tipos de investigación a que nos hemos referido anteriormente (investigaciones de problemas, exploratorias y experimentales) también es conveniente considerar las modalidades de investigación antropológica en relación con su incorporación al proyecto de salud pública. A este objeto, es conveniente tratar tres categorías generales de investigaciones: preliminares, durante el servicio y de evaluación.

\section{I nvestigaciones preliminares}

Son las que se llevan a cabo antes de iniciar las actividades de campo de un proyecto. Puede utilizarse de una manera exploratoria, con el objeto de obtener datos generales de carácter social y cultural y tratar de descubrir en la cultura y en la organización social puntos que pudieran presentar problemas en el desarrollo del proyecto. Puede igualmente concentrarse en un problema o ser de carácter experimental con el fin de resolver problemas específicos ya reconocidos con respecto a la sociedad de que se trata o para probar ciertas técnicas o métodos que el personal del proyecto desea utilizar en el curso de sus actividades. La investigación preliminar no puede concentrarse prácticamente en la organización, puesto que es improbable que la organización del proyecto exista antes de iniciar sus trabajos. Sin embargo, un antropólogo con experiencia puede contribuir a que se eviten problemas que más tarde pudieran plantearse debido a una estructura inadecuada de la organización.

El principal objeto de la investigación preliminar es evitar que se incurra en errores ostensibles desde el comienzo. El médico de salud pública, la enfermera o el ingeniero sanitario tienen que afrontar muchos problemas técnicos en el curso del planeamiento y desarrollo de un proyecto, Dados los muchos y muy importantes factores que intervienen, no supone descrédito para ellos si en ese planeamiento no tienen en cuenta los factores relativos a las diferencias culturales, relaciones humanas $u$ otros aspectos de la competencia de los especialistas de las ciencias sociales. Pero esos factores pueden tener importancia. Citaremos un solo ejemplo a este respecto. El autor ha tenido ocasión de examinar cierto número de planes iniciales de proyectos en los que los encargados de prepararlos habían tenido debidamente en cuenta las últimas estadísticas disponibles sobre la densidad de población del respectivo país. Sin embargo, en ningún caso se hizo observar que la cifra de la densidad estaba basada en la totalidad del territorio nacional y que, de hecho, en algunos casos incluso hasta la mitad de dicho territorio no estaba prácticamente ocupado. Como detalle ilustrativo, cabe señalar que aproximadamente el $94 \%$ de la población de Nicaragua 
está radicada en un poco más de la mitad del territorio nacional. Una cifra de densidad de población basada en la totalidad del territorio conduciría a conclusiones erróneas. Además, la cifra de la densidad nada indica respecto a la dispersión relativa de la población. ¿Está concentrada en las ciudades o esparcida en el campo, viviendo en granjas aisladas?

En condiciones ordinarias, los encargados de planear un programa de salud pública no disponen a menudo del tiempo necesario para realizar encuestas críticas sobre las características de la población a que se destina. También con frecuencia no están suficientemente familiarizados con el tipo de información disponible y que podría ser de suma importancia en su labor de planeamiento. Los servicios de un antropólogo pueden permitir que el planeamiento preliminar sea más realista y evitar que se planteen problemas debido a que los especialistas de salud pública no pueden reunir y analizar el volumen de información que ha de tenerse en cuenta

\section{I nvestigaciones durante el servicio}

Comprenden, como el término indica, las investigaciones llevadas a cabo durante el curso de un proyecto. La investigación más útil en este aspecto es, probablemente, la que se concentra en determinados problemas o es de carácter experimental. La fase media de un proyecto no es el momento oportuno para realizar extensos estudios exploratorios. Las investigaciones durante el servicio son sumamente útiles en materia de problemas de estructura orgánica y de las relaciones entre la organización y la sociedad. La investigación realizada durante el curso de un proyecto puede descubrir puntos de deficiente funcionamiento del mismo y poner en evidencia causas ocultas de dificultades. La investigación durante el servicio puede igualmente concentrarse en la sociedad para la solución de problemas que se presentan una vez emprendido el proyecto y en la ejecución de trabajos experimentales. En la práctica, la mayor parte del trabajo antropológico que se ha llevado a cabo hasta la fecha en proyectos de salud pública ha sido de este tipo.

\section{I nvestigación de evaluación}

Es, como su nombre denota, la investigación encaminada a evaluar alguna actividad, fase o trabajo, técnica o método. La investigación de evaluación puede llevarse a cabo en el curso de un proyecto o una vez concluido, pero difiere de la investigación durante el servicio en que tiene por objeto evaluar el relativo éxito o fracaso de algo que ya se ha realizado, Es en este sentido, una investigación de problemas. En razón de su naturaleza, debe llevarse a cabo una vez iniciada la actividad en cuestión, pero puede planearse e incluso iniciar algunas de sus fases antes de que comience el proyecto. El autor no conoce caso alguno, en su experiencia en la América Latina, en que en un proyecto relativo a cambio de hábitos de cualquier importancia, se emprendieran estudios preliminares acerca de los hábitos a que se refería el proyecto, y después se llevan a cabo investigaciones periódicas y una final con el objeto de determinar el grado de éxito alcanzado. Esto se ha hecho en situaciones experimentales, pero rara vez y de manera continua en proyectos en gran escala o de largo alcance. Desgraciadamente, algunos trabajadores de salud pública tienen la sensación de que el volumen de trabajo realizado en una actividad determinada refleja la eficacia de la misma. Como ejemplo de ello, cabe citar la evaluación que algunos educadores sanitarios establecen de su trabajo: el metraje de películas proyectadas al mes, los galones de gasolina utilizados en los viajes, el número de kilómetros recorridos en las visitas, el de personas que trabajan en las actividades de campo, el de reuniones celebradas con miembros de la colectividad, el de folletos publicados, etc. Todos estos elementos constituyen datos de actividad, pero ninguno 
de ellos indica en qué medida se ha logrado cambiar los hábitos de la población objeto de todos estos esfuerzos.

El término "evaluación" se ha popularizado desde hace algunos años en los medios de la salud pública, pero no en el sentido de investigación de evaluación. Si un administrador considera que el conservar su puesto depende de la obtención de resultados satisfactorios, no se sentirá inclinado a estimular una investigación que patentice que tales resultados no se han alcanzado. Por otra parte, resulta muy difícil a una persona o equipo que se incorpora a un proyecto en las últimas o última fase del mismo, tratar de establecer una evaluación cuando no se han hecho estudios preliminares en la materia. Muchas veces, la evaluación ha sido de esa naturaleza y los administradores se han mostrado, con razón, poco inclinados a que personas no familiarizadas con la situación local emitieran juicios sobre ella. Cuando hablamos de investigación de evaluación, no nos referimos a los comités de investigación, sino a la que se planea ordinariamente como parte integrante de un proyecto con el objeto de que el personal del mismo pueda saber en qué medida su propio trabajo está rindiendo los resultados perseguidos. No es la capacidad de los individuos (que es una cuestión administrativa) lo que se evalúa, sino la efectividad, técnicas y métodos que se aplican en el proyecto.

Hemos examinado en forma detallada la cuestión de la investigación en las actividades de proyectos, en razón de que, durante los últimos años, ha sido el campo donde las aportaciones antropológicas han sido particularmente notables y en el que, al parecer, la antropología puede hacer en el futuro una de sus mayores contribuciones.

\section{LOS ANTROPÓLOGOS COMO CONSULTORES}

Otra función que los antropólogos han desempeñado en relación con los proyectos de salud pública es la de los consultores o asesores. Es una de las funciones más antiguas del antropólogo en el campo de la salud pública y tal vez una de las que peor se han utilizado. El autor se ha referido en otro trabajo (Human Organization, Vol. 12, No. 2, pág. 12) a la preocupación que le producen los antropólogos profesionales que nunca han participado en la labor de aplicación en el campo, y que, no obstante, están siempre dispuestos a ofrecer asesoramiento, basándose en sus conocimientos generales. Sin embargo, no nos referimos aquí a esos consultores teóricos, sino a los antropólogos asignados por una organización de salud pública para actuar como consultores en proyectos específicos.

Para que los servicios de consulta sean efectivos se precisan dos requisitos esenciales: que haya alguien a quien consultar y algo que consultar. Aunque esto parece sencillo, resume los problemas fundamentales que se plantean al tratar de utilizar los servicios de consulta antropológica en el estado actual del desarrollo de las actividades de salud pública. Hay muchas personas a las que no se les ocurriría recurrir a los servicios de un antropólogo como consultor y muchas de ellas no sabrían qué discutir con él si se presentara el caso. Por otra parte, se observa creciente interés entre algunos administradores en utilizar los servicios de un antropólogo durante períodos limitados en cursos de adiestramiento o para la ejecución de trabajos concretos de investigación.

Teniendo en cuenta los problemas económicos que representa el mantenimiento de un consultor cuando no hay ocasión para utilizar sus servicios, y por otra parte, el valor reconocido de los servicios de los antropólogos en proyectos de campo cuando los administradores están percatados de su utilidad, deberían establecerse unas 
normas generales que permitieran el máximo uso de los antropólogos con el mínimo de costo.

Para contar con esos servicios de consulta, sería conveniente que las organizaciones de salud pública comenzaran a establecer una lista de posibles consultores a corto plazo en proyectos en que no se dispone o no se necesita de los servicios de un antropólogo empleado a tiempo completo. Esa lista debiera comprender personas que han trabajado durante algún tiempo en las regiones en que van a ser utilizadas. Además debiera fijarse una remuneración uniforme. Habría que informar a los antropólogos que han demostrado interés en las actividades de salud pública o que posean alguna experiencia en labor similar de aplicación, de las condiciones de esos nombramientos de consultores, y preguntarles si estarían dispuestos a actuar de tales en el caso de que se requieran sus servicios. Contando con esa lista, se podrían obtener servicios de consulta de personas expertas por períodos cortos y a breve plazo.

Cabe añadir, para completar este aspecto, que el empleo como consultor de un antropólogo clasificado es una categoría administrativa superior, puede ser a veces de sumo valor, si bien se ha hecho un uso relativamente escaso de tales consultores. Hay que señalar, de pasada, que en México los antropólogos sirven específicamente como administradores en proyectos generales que abarcan la salud pública entre sus actividades. Se menciona esto para recalcar que, en algunos lugares, se concede tanto valor a la aportación del antropólogo en la esfera administrativa, que incluso se le asigna el cargo de administrador.

\section{LA UTI LIZACIÓN DE ESPECI ALI STAS EN CIENCI AS SOCI ALES EN PROGRAMAS DE ADIESTRAMI ENTO}

Hemos mencionado anteriormente algunos de los problemas que se plantean al pretender presentar la antropología a los administradores de salud pública. Ahora vamos a ocuparnos de otra fase en la formación de personal de salud pública, con especial referencia a los países latinoamericanos que en la actualidad llevan a cabo programas encaminados a mejorar sus respectivos servicios de salud pública. Se recordará que, en relación con la utilización de un antropólogo como investigador en proyectos específicos, se mencionó que buena parte de los trabajos de investigación podían llevarse a cabo por personal especializado del proyecto, si contaba con la debida preparación. La formación de personal de proyectos para esta actividad presenta una serie de problemas que requieren solución.

Teóricamente, todos aquellos que desempeñan cargos en el campo de la salud pública cuyas funciones impliquen la adopción de decisiones respecto a cambios de hábitos de la población, debieran tener una formación bastante buena en ciencias sociales. Esta preparación no es simplemente para que estén mejores condiciones de llevar a cabo su trabajo de un modo inteligente, sino, lo que es todavía más importante, para que se percaten mejor de lo que hacen cuando deciden, por una razón u otra, cambiar los hábitos de otras personas. Bien pensado, es bastante presunción por parte de una persona decir a otra que no le gusta su manera de vida y que va a iniciar un proyecto para cambiarla, y esto es precisamente lo que hacen los trabajadores de salud pública. Dicen que los habitantes de determinada población aparecen muy sucios, no comen bien, tienen hábitos antihigiénicos, o algo parecido y que ellos (el personal de salud pública) se propone cambiar tales hábitos. No es preciso mencionar aquí las razones que justifican este proceder, puesto que se supone que todos los trabajadores de salud pública están debidamente impuestos de ellas. Lo que se necesita es más bien moderar ese arraigado prejuicio de salud pública. 
Además de las personas que ocupan puestos en los que han de adoptar decisiones (y para las que el conocimiento de lo que pudiéramos llamar a la filosofía de la antropología puede resultar ventajoso) el personal encargado de la ejecución práctica de los proyectos y los equipos ordinarios de salud pública pueden beneficiarse en gran medida del conocimiento de las temáticas de estudio y análisis aplicadas en algunas de las ciencias sociales. El campo de actividad del autor en esta materia ha sido, hasta la fecha, Centro América y, por tanto, las observaciones que figuran a continuación reflejan específicamente los problemas que ha encontrado en dicha región. Cree que esos problemas pueden muy bien plantearse en otras regiones y, por consiguiente, es probable que constituyan, hasta cierto punto, ejemplos típicos de las dificultades que habrán de encontrarse en la enseñanza de la antropología y de las ciencias sociales a los trabajadores de salud pública en otras partes.

Muchos de los trabajadores de salud pública con los que el autor ha colaborado, poseían uno de estos dos tipos de formación educativa: algunos tenían una cultura muy limitada, que a veces no pasaba de la enseñanza primaria; y otros habían sido objeto de un buen adiestramiento, pero su formación se inclinaba bastante hacia métodos de pensar especulativos, formales y no empíricos. Cada una de estas formaciones presenta sus problemas. Con respecto a las personas que poseen insuficiente formación, el principal problema es de la complejidad de las ciencias sociales. Los campos de la antropología, de la sociología y otros afines descansan hoy día en una serie de teorías en proceso de evolución, y la formación de personal profesional en los diversos campos se lleva a cabo principalmente una vez terminados los estudios de licenciatura. Las personas que estudian para ejercer en uno de los campos específicos, no solo cuentan con una enseñanza primaria y secundaria, sino que además han completado sus cuatro años de estudios universitarios. Gran parte de lo que aprenden en los cursos de especialización de ciencias sociales tiene sentido para ellos porque anteriormente han sido objeto de una formación liberal. Su estudio de la física les ayuda a comprender los conceptos de espacio, tiempo y movimiento; el estudio de la astronomía les ayuda a comprender el concepto de infinito; el de la filosofía y de las matemáticas les permite comprender las nociones de empirismo, racionalismo, lógica y filosofía de la ciencia; su estudio de la psicología les ayuda a comprender las fuerzas que operan en el cuerpo humano; su estudio de la historia y la arqueología les ayuda a ver el pasado como antecedente del presente; su estudio de la geografía, de la zoología y de la botánica les ayuda a comprender la extensa complejidad de la tierra y sus seres vivientes; su estudio de diversas ciencias les proporciona la formación necesaria para establecer conclusiones, aptitud sumamente importante; etc. Cada estudiante graduado aporta a su especialización una amplia serie de conceptos que le permiten comprender los utilizados en las ciencias sociales.

¿Qué relación tiene todo esto con la salud pública? Sencillamente la siguiente: muchos trabajadores de salud pública, en las categorías inferiores, carecen de la formación necesaria para comprender los conceptos expuestos en un curso breve de alguna especialidad de las ciencias sociales. Muchas veces, con el fin de dictar un curso que tuviera sentido para los estudiantes, el autor se ha visto precisado a remontarse tanto a los conceptos y puntos de vista fundamentales que no le fue posible exponer debidamente en el breve tiempo disponible, la mayoría de las ideas que hubieran sido más beneficiosas.

En relación con el personal de salud pública que posee adecuada o amplia formación, el problema es notablemente diferente pero no menos difícil. Mientras que la persona de escasa instrucción no tiene conocimientos de los conceptos básicos que le permitan comprender buena parte de las ciencias sociales, el hombre muestra a menudo una notable falta de perspectiva científica ante situaciones sociales. En la experiencia del autor, hubo un factor que constituyó un obstáculo principal en la enseñanza de las técnicas y métodos de las ciencias sociales: muy pocas personas a las que enseñó eran capaces de establecer una conclusión que estuviera basada en datos específicos. 
En ocasiones, teniendo ante ellos una serie de datos escritos ( $\mathrm{y}$ a menudo eran datos que ellos mismos habían recopilado), no establecieron una conclusión que tuviera relación alguna con los datos obtenidos. Cabe señalar que, en algunos casos, esas personas poseían una educación más amplia y completa y más años de experiencia que el autor. Pero se puso de manifiesto el defecto, sumamente grave, que representaba su propia seguridad respecto a cuál sería la conclusión sin que ni siquiera consideraran necesario reunir los datos pertinentes. La deducción de conclusiones, como cualquier otra labor que se trata de hacer de un modo sistemático, exige experiencia y práctica; no hay nada mágico a este respecto y no se precisa ser un genio. Pero requiere formación y además, que se haga.

Por supuesto, la mayoría del personal de salud pública puede establecer conclusiones basadas en hechos; sin embargo, a menudo no lo hacen en cuestiones sociales y culturales. Del mismo modo, no se trata de si el personal insuficientemente culto de las categorías bajas de salud pública es o no capaz de aprender los elementos fundamentales de la antropología y de las otras ciencias sociales, sino que se trata de una cuestión práctica, es decir, que carecen inicialmente de los conocimientos precisos para atender con provecho cursos breves en ciencias sociales.

La antropología y las ciencias sociales ofrecen dos cosas específicas al trabajador de salud pública: un punto de vista que le facilitará el conocimiento de la población en que trabaja, y algunas técnicas y métodos específicos para hallar la solución a problemas concretos. Resulta difícil decir cuál de estas dos cosas es la más importante, pero sin duda alguna la segunda es la que con más frecuencia se solicita. Los administradores de proyectos de campo han pedido de vez en cuando el autor de este trabajo que diera una disertación, unas pocas conferencias o un curso breve "para ayudar al personal de campo". Si bien uno comprende perfectamente el deseo de proporcionar al personal de campo una introducción a la antropología, trata de evitar tales solicitudes, ya que estima que esos esfuerzos son prácticamente vanos.

En ciertas condiciones que el autor considera deben concurrir para que un curso de antropología o sobre algunos aspectos de esta ciencia resulte de utilidad al personal de campo de los programas de salud pública:

En primer lugar, el número de horas del curso y el período de tiempo en que se distribuyan han de decidirse de acuerdo con la formación general que el personal tenga. Esto supone que no podemos determinar a priori si se necesitarán 10 ó 50 horas de clase, o si el curso ha de durar un mes o un año. Sin embargo, salvo en la enseñanza de técnicas específicas a personas capaces de comprender la teoría en que se basan, pueden excluirse los cursos breves de una o dos semanas de duración.

En segundo lugar, la materia de estudio en un curso de esa clase se debe preparar cuidadosamente de acuerdo con el administrador del proyecto de adiestramiento, con el objeto de que los aspectos específicos de las ciencias sociales que se enseñen sean de verdadera utilidad. La antropología es una amplia disciplina en sí misma y es sólo una de las ciencias sociales. La elección de las materias específicas que han de incluirse no es un problema que pueda resolverse fácilmente a priori. Esto supone que la persona que enseñe el curso debe disponer de un período de preparación a fin de que pueda sondear la formación general de los estudiantes y las características de los problemas que habrán de afrontar en sus actividades.

En tercer término, es indudable que la capacitación sobre el terreno es un aspecto en el que la antropología puede hacer una aportación sumamente importante, pero para actuar con sentido realista no se tratará de proporcionar experiencia práctica sin la correspondiente enseñanza de la teoría, acompañada de discusiones y lecturas sobre 
la materia. Es lo mismo que si se da a un niño una serie de piezas y se le dice que construya un rascacielos de juguete, sin saber lo que es un rascacielos.

El cumplimiento de esas condiciones puede contribuir en gran medida al mayor éxito de la preparación en antropología del personal de proyectos. Un forma de alcanzar esas condiciones es la de asignar un especialista en ciencias sociales a un proyecto por un período limitado, pero dándole tiempo suficiente por anticipado para que conozca las necesidades de los estudiantes, prepare un curso de acuerdo con esas necesidades y en consulta con los administradores del proyecto, y coordine el curso con el adiestramiento durante el servicio que se facilite al personal. La asignación de un antropólogo a un proyecto de esa clase ofrece la ventaja práctica de que le permite familiarizarse mejor con el medio cultural en el que el personal del proyecto ha de trabajar, dándole también la oportunidad de prestar mayor atención a aquellas materias de estudio que les permitan comprender mejor la situación en que se encuentran. Ahora bien, debe recordarse que si se asigna un especialista en ciencias sociales a un proyecto para desempeñar esas funciones, el período dedicado a la investigación para el proyecto quedará considerablemente reducido.

El adiestramiento en el curso de la ejecución del proyecto beneficiará en gran medida al personal interesado, pero no debe creerse que puede reemplazar el adiestramiento facilitado en las escuelas de salud pública de enfermería, etc., en las que el personal de salud pública recibe su preparación intensa en los problemas de salud pública. Hasta que muchos de esos problemas sean considerados como problemas de relaciones humanas y de cambios de hábitos, seguirán presentando dificultades que no se pueden resolver por los métodos ordinarios de salud pública. La preparación en tales materias puede facilitarse mejor al mismo tiempo que el interesado aprende lo que son esos problemas desde el punto de vista de su respectiva profesión.

\section{CONCLUSI ONES GENERALES}

Este trabajo tiene por objeto describir en detalle algunos conocimientos obtenidos sobre la utilización efectiva de la antropología y de las ciencias sociales en el campo de la salud pública. Refleja el punto de vista de un especialista que actúa en Centro América y con las preocupaciones particulares de una persona que trabaja al servicio de una organización internacional. No se ha tratado de describir en detalle la forma en que opera la antropología o en qué consiste; estas materias han sido tratadas en otros estudios por varios trabajadores en el campo.

Basándose en el informe publicado por George Foster en 1951, se puede establecer una comparación entre nuestros puntos de vista actuales y los expresados hace tres años. Como resumen, mencionaremos los puntos establecidos en este informe juntamente con las recomendaciones generales formuladas originalmente por Foster.

1. Los administradores de programas de salud pública deben tener cierto conocimiento del medio cultural en que trabajan. Para ello, es evidente que los propios administradores necesitan preparación en los principios de las ciencias sociales y que han de hacer estudios del medio cultural en que trabajan. El método más efectivo para poner de manifiesto el valor de esos conocimientos es, probablemente, el de contar con antropólogos profesionales u otros especialistas en ciencias sociales adscritos a los proyectos. En los últimos años, algunas escuelas de salud pública han establecido cursos en antropología y relaciones humanas, pero esto surtirá poco efecto en aquellos que hace tiempo se graduaron en dichas escuelas y, además todavía hay muchas que no cuentan con tales materias en su plan de 
estudios. Para que los administradores puedan adquirir conocimientos fundamentales en campos poco conocidos, es preciso llevar a cabo estudios exploratorios.

2. Deben asignarse especialistas en ciencias sociales a los equipos de campo en actividades de salud pública. El hecho de que sea o no necesario asignar un antropólogo u otro especialista en ciencias sociales a los equipos de campo dedicados a actividades de salud pública depende de una variedad de factores, a saber: que el administrador desee contar con sus servicios; las características del proyecto propuesto; las diferencias que existan entre el personal del proyecto y la población de que se trata; la efectividad de la estructura orgánica del proyecto; si antes se han realizado o no investigaciones relativas al proyecto; el grado de conocimiento que el personal del proyecto posea acerca de los conceptos y técnicas utilizadas en materia de relaciones humanas, etc. Ahora bien; es igualmente evidente que el antropólogo puede rendir mayores servicios si actúa como miembro de un equipo de campo. De acuerdo con las necesidades del proyecto, puede ejercer cualquiera de las tres funciones siguientes a todas ellas: investigador, consultor y profesor. Puesto que su formación le capacita específicamente para la investigación, hemos descrito ciertas fases y aspectos de la investigación que puede llevar a cabo ventajosamente como miembro de un equipo de campo o del personal del proyecto.

3. Es preciso continuar los estudios básicos, ya que en ellos se fundan los conocimientos especializados. Aun cuando, como señaló Foster, la realización de estudios básicos como fundamento para las actividades especializadas en salud pública no es necesariamente función que corresponda a un proyecto de salud pública, tales conocimientos básicos constituyen, no obstante, una necesidad esencial. Por consiguiente, a los programas de salud pública corresponde la responsabilidad de promover los estudios necesarios de exploración en las regiones donde todavía no se han llevado a cabo. Esos estudios pueden realizarse como investigaciones preliminares para programas en proyecto.

En relación con los tres puntos antes mencionados, podemos decir, en general, que desde 1951 se han hecho progresos en la preparación de administradores y en la promoción de estudios fundamentales. Ahora bien; considerando a la América Latina como un todo, hay pocas pruebas de que se hayan obtenido adelantos importantes en el empleo de antropólogos donde pueden ser de mayor utilidad, concretamente, en proyectos de salud pública en la América Latina, tres han renunciado y no han sido reemplazados y uno se ha dedicado a estudios fundamentales. En 1952, el Instituto de Asuntos Interamericanos contaba con cuatro antropólogos; ahora, según nuestra información, sólo cuenta con dos. La Organización Mundial de la Salud emplea solamente en la actualidad un antropólogo en el Hemisferio Occidental. Los antropólogos han tenido mayor participación en ciertos proyectos de la Organización de los Estados Americanos, Organización Internacional del Trabajo, UNESCO, y Naciones Unidas que en cualquiera de las organizaciones específicas de salud pública.

Aparte de los diversos puntos descritos por Foster, hay otros dos que han quedado aclarados.

4. El personal dedicado a la ejecución de proyectos puede beneficiarse del adiestramiento en los conceptos y técnicas de la antropología. El autor de este trabajo, en su calidad de antropólogo al servicio de la Organización Mundial de la Salud, así como anteriormente, recibió solicitudes para dictar cursos a personal de proyectos. Teniendo en cuenta que hay muchos estudiantes que el propio personal de proyectos puede llevar a cabo si cuenta con la adecuada preparación, esa labor puede ser de suma utilidad. Ahora bien; como se ha indicado ya, ese adiestramiento puede dar resultados satisfactorios únicamente si concurren determinadas condiciones, y 
además hay limitaciones a lo que puede lograrse con personal de escasa formación y educación liberal. Debe prestarse mayor consideración a la organización y objetivos de tales programas de adiestramiento, tanto en términos generales como en relación con proyectos específicos.

5. Los consultores antropólogos en las esferas intermedias y superiores de la administración y planeamiento. El autor no conoce experiencias o conclusiones publicadas respecto a este tipo de servicios de consulta. Sin embargo, basándose en sus propias impresiones, estima que una vez se conceda suficiente valor al punto de vista antropológico en las altas esferas de la administración, el antropólogo puede desempeñar un papel valioso como consultor permanente de esas esferas.

En los tres años transcurridos desde la aparición del informe de Foster, se ha obtenido algún progreso práctico en la utilización efectiva de la antropología. Además de haberse reconocido su utilidad en los programas de adiestramiento de salud pública, tanto en las escuelas como en el campo, se han promovido estudios básicos sobre la materia. Sin embargo, cuando se considera desde el punto de vista del cuadro general de la salud pública y de la contribución efectiva que la antropología puede aportar, el progreso no ha sido amplio ni espectacular. Ha habido muchas expresiones verbales de interés; personas que trabajan en el campo de la salud pública o fuera de él han publicado ocasionalmente artículos sobre la materia, pero las nuevas medidas prácticas adoptadas han sido relativamente escasas.

El autor no está en condiciones de poder determinar la significación de todo ello. Por supuesto, en gran parte puede imputarse al hecho de que las organizaciones de salud pública son burocráticas, a lo cual puede achacarse, en alguna medida, la lentitud en esta materia. Ahora bien, muchas veces se culpa indebidamente a la burocracia de la mala disposición de los individuos. El autor tiene la impresión de que algunos administradores están esperando a que alguien promueva activamente la utilización de la antropología y cometa errores que inevitablemente han de ocurrir en las fases iniciales de una actividad semejante. Mientras tanto, ya se utiliza la antropología en grado suficiente para permitirles decir que ellos figuraron entre los primeros que fomentaron su aplicación. Que esto pueda atribuirse enteramente a la falta de conocimientos a que nos hemos referido en este trabajo, o bien a otros factores (tales como el letargo individual o la política de organización) dependen en gran medida de cada caso en particular.

Es evidente, sin embargo, que la mayoría de las organizaciones de salud pública carecen de una política definida respecto a la utilización de la antropología. Ninguna de ellas se ha comprometido a utilizarla, a no utilizarla, o ni tan siquiera a someterla a una serie de pruebas efectivas. Ninguna ha llegado a decir: "La antropología ha demostrado cierta utilidad; procedamos a ensayarla en ciertos campos limitados y durante determinado período de tiempo con el objeto de determinar esa utilidad con mayor precisión". El presente trabajo ha sido escrito, no meramente con el propósito de precisar esa utilidad y contribuir a determinar el estado actual de la antropología en el campo de la salud pública, sino además para señalar esa falta de política respecto a la utilización de las ciencias sociales en la salud pública. Esa falta se refleja no sólo entre el personal de salud pública, sino también entre algunos especialistas en ciencias sociales que han trabajado en proyectos de salud pública. Los antropólogos no tratan de convencer a las esferas responsables de la salud pública de la utilidad de sus servicios, pero a menudo se les coloca aparentemente en la situación de tener que hacerlo. No cabe duda que ello ha contribuido a que algunos antropólogos perdieran la paciencia con las actividades de salud pública y volvieran a trabajar completamente dentro de su propia disciplina. En realidad, sería una desgracia que 
los medios de la salud pública, por carecer incluso de una política inicial, alejaran a esos colaboradores en lugar de estimular su ayuda.

* El autor expresa su agradecimiento a las siguientes personas que leyeron este manuscrito y formularon críticas sobre el mismo: Dres. George Foster, Guillermo Samamé, J.L. García Gutiérrez, Nevin S. Scrimshaw, Stanford F. Farnsworth y Sr. Norman Craig, y asume la responsabilidad plena de las opiniones expresadas en este estudio, las cuales no son necesariamente las de las personas antes mencionadas. Este artículo aparece en inglés en Human Organization, Vol. 13, No. 4, 1955.

** George Foster, al leer este párrafo, comentó que probablemente era demasiado pronto para definir el grado de utilidad posible de las ciencias sociales en el campo de la salud pública. Sin embargo, el autor considera que es conveniente estudiar este problema como un medio de aclarar nuestras ideas. Este párrafo, como todo el artículo, no debe ser considerado como una exposición de hechos, sino que trata de suscitar ulteriores definiciones y aclaraciones. 